\title{
Article \\ Symmetry and Symmetry Breaking in Physics: From Geometry to Topology
}

\author{
Luciano Boi (D)
}

Citation: Boi, L. Symmetry and Symmetry Breaking in Physics: From Geometry to Topology. Symmetry 2021, 13, 2100. https://doi.org/ $10.3390 /$ sym 13112100

Academic Editors: Ignazio Licata and Roberto Passante

Received: 24 September 2021

Accepted: 20 October 2021

Published: 5 November 2021

Publisher's Note: MDPI stays neutral with regard to jurisdictional claims in published maps and institutional affiliations.

Copyright: (C) 2021 by the author. Licensee MDPI, Basel, Switzerland. This article is an open access article distributed under the terms and conditions of the Creative Commons Attribution (CC BY) license (https:/ / creativecommons.org/licenses/by/ $4.0 /)$.
École des Hautes Études en Sciences Sociales, Centre de Mathématique (CAMS), 54, bd Raspail, 75006 Paris, France; boi.luciano@ehess.fr

\begin{abstract}
Symmetry (and group theory) is a fundamental principle of theoretical physics. Finite symmetries, continuous symmetries of compact groups, and infinite-dimensional representations of noncompact Lie groups are at the core of solid physics, particle physics, and quantum physics, respectively. The latter groups now play an important role in many branches of mathematics. In more recent years, we have been faced with the impact of topological quantum field theory (TQFT). Topology and symmetry have deep connections, but topology is inherently broader and more complex. While the presence of symmetry in physical phenomena imposes strong constraints, topology seems to be related to low-energy states and is very likely to provide information about the different dynamical trajectories and patterns that particles can follow. For example, regarding the relationship of topology to low-energy states, Hodge's theory of harmonic forms shows that the zero-energy states (for differential forms) correspond to the cohomology. Regarding the relationship of topology to particle trajectories, a topological knot can be seen as an orbit with complex properties in spacetime. The various deformations or embeddings of the knot, performed in low or high dimensions, allow defining different equivalence classes or topological types, and interestingly, it is possible from these types to study the symmetries associated with the deformations and their changes. More specifically, in the present work, we address two issues: first, that quantum geometry deforms classical geometry, and that this topological deformation may produce physical effects that are specific to the quantum physics scale; and second, that mirror symmetry and the phenomenon of topological change are closely related. This paper was aimed at understanding the conceptual and physical significance of this connection.
\end{abstract}

Keywords: symmetry; breaking symmetry; geometry; topology; physics; spacetime; deformation; dynamics

\section{Introductory Remarks}

"The history of physics is not only a sequence of experimental discoveries and observations, followed by their mathematical description; it is also a history of concepts." W. Heisenberg.

The concept of symmetry has attracted philosophers and mathematicians since the ancient time [1]. The contributions of Plato [2] and Kepler have been extremely fruitful, and their ideas concerning the key function of symmetry in the ordering of the real world are still relevant today. The work of Kepler deserves special comment. He searched for the constitution of physical world. He indubitably believed that the architecture of the cosmos must fulfill certain symmetries. The key to this architecture resided not in numbers but in geometry. Whereas for Galilei, elliptical orbits broke the symmetry of the cosmos [3], for Kepler, the consideration of these orbits paved the way to the discovery of "hidden" deeper symmetries. For him, the veracity of elliptical orbits was a consequence of necessary physical conditions and of laws of harmony [4,5]. With the scientific revolution in the sixth century, the concept of symmetry became the core of a new vision of the universe and of nature [6-9]. This conception strengthened even more with the discovery of relativity 
theory, both special and general, at the beginning of the twentieth century. However, later on, the fundamental role played by the phenomenon of spontaneous symmetry breaking (SSB) in the organization and evolution of natural and living phenomena was recognized (we refer to [10-17] for a detailed presentation of the subject).

A physical system is symmetric if the system remains unchanged when some transformations are applied to it. The key concept behind the fundamental equations of physics is conservation, as defined in the "conservation laws". For an isolated or quasi-isolated system (i.e., in absence of external forces), the most basic physical conserved quantities are energy (which transforms remaining constants), linear momentum (i.e., the product of mass and vector velocity), angular momentum (of an object in circular motion), and electric charge (a very important property of matter when affected by an electromagnetic field). It must be specified, however, that the momentum for a massless particle, like photon, is not the product of the mass and the velocity (which is 0 ). This is quite relevant for the theory of special relativity, wherein the concept of relative mass (defined by Einstein in 1905) and the related mass-energy equivalence explain why gravity may have an influence on light (photons). Special relativity proved that a photon does have relativistic mass proportional to its momentum. Furthermore, De Broglie's relation, concerning quantum theoretical wave-particle duality, yields (assuming that $m$ is relativistic mass) the important result that photons have mass inversely proportional to their wavelength.

The symmetry principle plays a fundamental role in mathematics and in the natural sciences, particularly in physics. According to this principle, the group of symmetries governing the causes of a physical system form a subgroup belonging to the group of symmetries resulting from the effects. Differently stated, the effects can have a larger group of symmetries than the causes. However, these principles, in many situations, are contradicted by the phenomenon of "spontaneous symmetry breaking", which means that there are physical systems where the effects simply have less symmetry than the cause. In other words, the actual physical world is less symmetric than the fundamental equations of physics. Mathematically, this can be expressed by saying that the symmetries that these equations obey are more general than the symmetries of the physical solutions of the equations when spontaneous symmetry breaking occurs. This claim should, however, be completed by saying that the spontaneous breaking symmetry mechanism is often strictly connected with the dynamical rearrangement of symmetry. For example, in quantum field theory, the symmetry at level of asymptotic fields is the group contraction of the symmetry at level of interacting fields (same number of generators). For more details, see [18-21].

Spontaneous symmetry breaking occurs in superconductivity, the electroweak interaction, early universe formation (as predicted by inflationary cosmology), and many other physical contexts. Well-known examples of this mechanism of breaking-symmetry are the phase transition from a liquid to a crystal and the violation by the "standard model" of the symmetry between matter and antimatter.

\section{The Groups of Minkowskian and Einsteinian Spacetimes}

Let us now present the most important conceptions of space-time by comparing them with respect to their respective groups of symmetries. The relation between Minkowskian and Einsteinian spacetimes mirrors that between Galilean and Newtonian spacetimes (see [22-24]). Thus, Minkowskian spacetime has a unique description (for example, its geodesic structure is the same as that of $R^{4}$, the set of time-like geodesics corresponding to geodesics in $R^{4}$ making an angle less than $45^{\circ}$ with some fixed direction), and it does not describe gravitation. It has a ten-parameter transitive symmetry group of motion, namely, the Poincaré (i.e., the inhomogeneous Lorentz) group. According to Stachel [25], "Any set of dynamical variables must form a representation of this group and obey dynamical equations that transform appropriately under it. More precisely, the set of dynamical variables at each point must form a representation space of the homogeneous Lorentz group. For fields, the dynamical equations must guarantee that these representations together form a representation of the inhomogeneous Lorentz (Poincaré) group. Theories 
obeying these constraints are said to be Lorentz- or Poincaré-invariant" (for a detailed presentation see [26]).

On the other hand, many Einsteinian spacetimes exist, corresponding to the various inequivalent gravitational fields (see [27]). As with the Newtonian spacetime, it is the tidal gravitational force that has physical significance, this being present when there is a gradient of the "gravitational acceleration field". This tidal force is described in terms of deviations of the intrinsic geodesic structure from that of Minkowskian spacetime, that is to say, by the curvature of the Riemann-Einstein spacetime. Einstein's field equations then described how this spacetime curvature is related to the density of matter-that is, of stress-energy-momentum (see below). Finally, the metric of spacetime must differ, in a gravitational field, from the ordinary flat spacetime form. In general relativity, one is led to look for a theory which agrees locally (approximately) with special relativity, but has, instead of the integrable, affine connection of Minkowski spacetime, a nonintegrable linear connection with curvature capable of representing the combined inertial-gravitational field.

The central feature of general relativity is that it furnished, for the first time, a unified description (and explanation) of space, time, and gravitation that is essentially geometrical in nature. Indeed, the gravitational field is represented by a (symmetric) connection $\Gamma$ (which is a geometrical object), and the equations relating the gravitational field to its material source (i.e., to matter) can be expressed as a relation between the contracted curvature tensor $R_{i j k}^{l}$ of $\Gamma$, so that the second law of dynamics can be formulated in terms of a covariant derivative with respect to $\Gamma$. Resulting from the first, another central feature of general relativity is the principle of general covariance, a constraint on the dynamical equations permitted by the theory. The symmetry group acting (locally) on the fourdimensional spacetime manifold, on which all physical fields are defined, consists of all diffeomorphisms (sufficiently smooth point transformations of the manifold); this group leaves invariant a general quadratic form (the metric) characterizing the manifold.

The influence of general relativity in twentieth-century mathematics has been very relevant. It gave a considerable boost to the growth of differential geometry as a mathematical subject, which developed into one of the most fruitful branches of mathematics, with applications in many other areas [28]. These developments immediately led, beginning in the 1920s, to generalizations by Levi-Civita, Schouten, Veblen, E. Cartan, and Eisenhart. It soon became clear that in the applications of Riemannian geometry to relativity, Levi-Civita parallelism and not the Riemann metric itself plays the crucial role. In his famous book Raum, Zeit, Materie, published in 1918, H. Weyl introduced the notion of an affine connection. This is a structure, not necessarily Riemannian, for which parallelism and covariant differentiation are defined with the desired properties. Weyl's affine connections are symmetric, or without torsion. A definitive treatment of affine connections, together with a generalization to connections with torsion, was given by Elie Cartan in his fundamental paper, "Sur les variétés à connexion affine et la théorie de la relativité générale" ([29]; see also [30]).

On this subject, the theoretical physicist Tullio Regge made the interesting following remarks [31]: "There is no difficulty in writing the modern (gauge) form of electromagnetism (with the compact group $S O(1)$ or $U(1)$ ) on a Riemannian manifold and it is possible to write, à la Cartan, general relativity as a $S O(3,1)$ gauge theory. Besides, it may be useful to recall that Cartan was largely responsible for the introduction of the concept of torsion in Physics (see [30,32]). Torsion remain a very interesting idea. We need to use it, even by just declaring it to vanish, if we want to write general relativity as a gauge theory in which all fields and not only the spin connection appears as gauge potentials. The interesting feature of general relativity is that the associate curvature of the vierbein, i.e., torsion, vanishes as a consequence of the variational principle of Hilbert-Einstein-Cartan. And in fact, the Lagrangian density is not invariant under all gauge transformations of the Poincare group but only under those of the Lorentz subgroup. Although nature has prepared the gauge potentials for the full group it ends up by requiring invariance under a subgroup only. A 
world with torsion would appear inescapable if we have around enough density of high spin particles which acts as sources, but this density seems at the moment well below the limit of observability. Regards the kind of space in which torsion is supposed to appear, one can remark that it would not be any more a Riemannian manifold or, rather, none of the Riemannian structures existing on the manifold would be directly related to Physics and the theory would not be a geometrical theory in the sense envisaged by Einstein. One could yet consider general relativity as $G L(4, \mathbf{R})$ theory with the Christoffel connection playing the role of a Yang-Mills potential. If the torsion vanishes it follows that the Christoffel symbol is symmetrical into the 2 lower indices whose role is however quite different. The first index is a $G L(4, \mathbf{R})$ gauge index, the second labels instead the differentials of spacetime. We may relate them because of the accidental and marvelous fact that the Jacobian group of derivatives on a differentiable manifold is isomorphic to $G L(4, \mathbf{R})$ and that we use the same indexing for differentials and vectors in $G L(4, \mathbf{R})$. Once the symmetry is established the theory becomes almost by definition geometrical. If there is no symmetry but we can control torsion by introducing suitable norms as bounds then we may still speak of an almost geometrical theory whose exact mathematical definition is still lacking."

Let us recall that, geometrically, an affine connection is a family of affine spaces, the fibers parametrized by a space, the base space, such that the family is locally trivial and that there is a law of "development" of the fibers along the curves of the base space that preserves the latter's linear properties. In the same vein, we can take Klein spaces as fibers and replace the general linear group with a Lie group acting on the Klein space, with a corresponding law of development. This structure was called by Cartan a generalized space ("espace généralisé"). In general, the connection is nonholonomic; that is, the development depends on the curve in the base space. In other words, the space does not return to its original position after being developed along a closed curve; the measure of the deviation is given by the curvature of the connection. Clearly, a Klein space is itself a generalized space, with curvature identically zero. From Cartan's viewpoint, Riemannian space is a generalized space with Euclidean spaces as fibers and provided with the Levi-Civita connection (this is one of the fundamental features of physical space according to general relativity). This settled a foundational issue in differential geometry, as we have now a notion that includes Klein spaces, Riemannian spaces, and generalization of both.

The development in what is commonly described as non-Riemannian geometry was accompanied by parallel development in general relativity. With special relativity for the electromagnetic field and general relativity for the gravitational field, there was clearly a need for a unified field theory in which the two could be combined. The first significant step toward this theory was taken by H. Weyl in 1918 [33] in his gauge theory. Weyl introduced a generalized space having as a group the group of homothetic transformations. It was found to be physically untenable in any non-quantum mechanics framework. It is now understood that his gauge group should not be the noncompact group of homothetic transformations but the compact circle group. Weyl's theory is in fact based on the geometry of a circle bundle over a Lorentzian manifold. Therefore, the linear differential form $\varphi$, subject to the gauge transformation, can be interpreted as defining a connection in the circle bundle, and the quadratic form $d s^{2}$ can remain unaltered. In other words, Weyl's quantum gauge theory deals with a circle bundle or a $U(1)$-bundle, that is, a complex Hermitian bundle of dimension one (for more details we refer to [34-37]).

According to Yang-Mills theory, the physical isotopic spin can be regarded essentially as a mathematical object, namely a connection on an SU(2)-bundle [38]. Thus, we have the first example of a gauge theory for which the group is non-Abelian (i.e., noncommutative) $[17,39]$. To a connection can be associated an "action" of the gauge group (see [40]). The action taking a minimum over an $S U(2)$-bundle is called an instanton. An instanton corresponds to a self-dual curvature $F_{A}$ (physically, the field strength tensor of gauge theory) of a principal bundle with connection $A$ (physically, the gauge potential). Self-dual instantons provide a solution of the Yang-Mills equation. Let the four-dimensional sphere $S^{4}$ be the compactified model of the space $R^{4}$; then there exists a topological quantum 
number $k$ (an integer), which determine up to an isomorphism the $S U(2)$-bundle. Let $M$ be a compact oriented four-dimensional Riemannian manifold, and $G$ a compact semisimple Lie group; then Atiyah, Hitchin, and Singer (1978) proved that there exist principal G-bundles $P \rightarrow M$ that admit smooth, irreducible self-dual connections over $S^{4}$. Let $P \rightarrow S^{4}$ be a principal G-bundle. A principal over $S^{4}$ with simple connected structure group is classified by an integer $k$, called the Pontrjagin index (for more details we refer to [41]).

In physical terms, this is the dimension of the space of instantons with topological quantum number $k>0$. Atiyah and Ward observed that the self-dual Yang-Mills fields fit well into the Penrose twistor program. They were able to translate the problem of finding all self-dual solutions into a problem of algebraic geometry: classifying holomorphic vector bundles on the projective 3-space. Using results related to such classification, it has been shown that one can then find all self-dual solutions. There is a relationship between general relativity and instantons, which can be explained as follows. The special orthogonal group $S O(4)$ is locally isomorphic to the product $S U(2) \times S U(2)$. In this way, connections over the $S U(2)$-bundle are uniquely determined through projection by a Riemannian metric defined on a four-dimensional manifold $(M, g)$. If connections are self-dual $\left(P-F_{A}=0\right)$ or anti-self-dual $\left(P+F_{A}=0\right)$, we have an Einstein manifold. Every self-dual connection is a Yang-Mills connection (for a complete treatment of the subject see [42]).

\section{Some Mathematical Facts and Results}

The concept of Riemannian structure presupposes that of tangent bundles over smooth manifolds. What is needed is to start with is a definition of tangent vectors at a point $x \in \mathbf{R}^{n}$ (tangent vectors are simply ordinary vectors attached to $x$ ). However, to make the definition applicable to any manifold (using local coordinates), it is better to change it by considering all possible maps and defining the equivalence of two such maps if and only if the first derivatives of the corresponding functions are equal (the derivatives along local coordinates $x_{1}, \ldots, x_{n}$ in $\mathbf{R}^{n}$ can serve as a coordinate system in the tangent space). Thus, an equivalence class with respect to this equivalence relation is called a tangent vector (at the point $x$ ). The set of all tangent vectors at a point $x$ is called the tangent space at this point. It turns out that the operations in the tangent space do not depend on the choice of coordinates in $\mathbf{R}^{n}$. The tangent space to a manifold $M$ at a point $x$ is defined similarly using an arbitrary local coordinate system and is denoted by $T_{x} M$. For a smooth manifold $M^{n}$, consider the set $T M^{n}$ consisting of all tangent vectors at all points of $M^{n}$, i.e., elements of $T M^{n}$ are pairs $(x, v)$ such that where $x \in M^{n}, v \in T_{x} M^{n}$. There is a natural way to introduce a topological space (and even a smooth manifold) structure on $T M^{n}$. Consider a cover of $M^{n}$ by charts $V_{\alpha}$. For each chart, the homeomorphism $\varphi_{\alpha}: V_{\alpha} \rightarrow U_{\alpha} \subset \mathbf{R}^{n}$ determines a one-to-one correspondence between $T V_{\alpha}$ and $T U_{\alpha}$. But $T U_{\alpha}=U_{\alpha} \times \mathbf{R}^{n}$, since tangent spaces at distinct points of $\mathbf{R}^{n}$ are identified by means of parallel translations. There is a natural structure of a smooth manifold on the set $T U_{\alpha}=U_{\alpha} \times \mathbf{R}^{n}$ (and, therefore, a topological space structure). This structure descends to $T V_{\alpha}$. On the intersections of charts $T U_{\alpha}$, the maps are compatible (i.e., the union of these charts is a smooth atlas on $T M^{n}$ ). The space $T M^{n}$, endowed with the natural projection $T M^{n} \rightarrow M^{n}$, is called the tangent bundle. Each fiber of this bundle is endowed with an $n$-dimensional vector space structure.

Definition 1. A Riemannian metric (or Riemannian structure) on $M^{n}$ is a smooth function $g$ : $T M^{n} \rightarrow \boldsymbol{R}$ (of class $C^{\infty}$ ) such that its restriction to any fiber $T_{x} M^{n}$ is a positive definite quadratic form $($ see $[43,44])$.

Any such form defines a symmetric bilinear form; therefore, any Riemannian structure defines an inner product on the tangent space at any point $x \in M^{n}$. A quadratic form allows one to define the length of any tangent vector; namely, the length $\left\|v_{x}\right\|$ of a vector $v_{x} \in T_{x} M^{n}$ is equal to $\sqrt{ } g\left(v_{x}\right)$.

Theorem 1. There is a Riemannian structure on any smooth manifold (for the proof, we refer the reader to $[44,45])$. 
We need here to recall another important fact of Riemannian geometry. Any Riemannian $n$-manifold has a Riemann curvature tensor $R_{i j k l}$ (working in an orthonormal basis for the tangent space at a point). This is skew symmetric in the indices (ij) and $(k l)$ and satisfies the Bianchi identity:

$$
R_{i j k l}+R_{i k l j}+R_{i l j k}=0
$$

The Riemann curvature tensor $R$ satisfies the following properties:

(a) (Symmetry) $R_{i j k l}=-R_{j i k l}, R_{i l j k}=-R_{i j k l}, R_{i j k l}=R_{k l i j}$;

(b) (first Bianchi identity) the sum of $R_{i j k l}$ over the cyclic permutation of any three indices vanishes;

(c) (second Bianchi identity) $R_{i j k l, h}+R_{i j l h, k}+R_{i j h k, l}=0$, where $R_{i j k l, h}=\left(\nabla_{\partial \mathrm{h}} R\right)_{i j k l}$.

(For more details on this subject and further development, we refer to [46-48]).

\section{The Geometric Sources of Gauge Theory: From Weyl to Yang-Mills}

In developing the theory of gravitation, Einstein started with the problem of accounting for the equality of inertial and gravitational mass. In order to answer to this question, it was necessary to admit nonlinear transformations of the four coordinates of spacetime. In Einstein's theory of gravitation, the laws of nature obey transformations (forming a continuous group) that occur regardless of the equations governing these laws. Neighboring points have the same coordinates, which fact corresponds to a topological order of points in space. General relativity rests upon this fundamental principle (see $[49,50])$. The relation between neighboring spacetime points in four dimensions must, in accordance with the principle of general relativity, admit certain arbitrary continuous transformations of the coordinates. The relation thus assumes the general form:

$$
d s^{2}=g_{i j} d x^{i} d x^{j} \text { where }\left(g_{i j}=g_{j i}\right)
$$

$g_{i j}$ are the functions of the coordinates $x^{i}$ such that $g=\left|g_{i j}\right| \neq 0$. They transform in a definite way if a continuous coordinate transformation is applied. The quadratic differential form (2) is called the Riemannian metric for $n$-dimensional Riemannian space, and $g_{i j}$ is called the metric tensor, which is a symmetric two-tensor obtained by symmetrizing the covariant derivative of the Riemannian space.

More extensively: Let $M$ be a manifold, and let $p$ be a point of $M$. Then, TM denotes the tangent bundle of $M$, and $T_{p} M$ is the tangent space at $p$. Similarly, $T^{*} M$ denotes the cotangent bundle of $\mathrm{M}$ and $T^{*}{ }_{p} M$ is the cotangent space at $p$. For any vector bundle $\mathcal{V}$ over $M, \Gamma(\mathcal{V})$ denotes the vector space of smooth sections of $\mathcal{V}$. A Riemannian metric on a smooth manifold $M$ is defined as a covariant symmetric two-tensor $(.,)_{p}, p \in M-$ a section of the symmetrized second tensor power of the tangent bundle-such that $(v, v)_{p}>0$ for all $v T_{p}(M)$. Differently stated, a Riemannian metric is a collection of (positive) inner products on each of the tangent spaces $T_{p}(M)$ such that if $X, Y$ are (smooth) vector fields, the function $(X, Y): M \rightarrow \mathbb{R}$, defined by taking the inner product at each point, is smooth.

According to the principle of equivalence between inertial and gravitational fields, the $g_{i j}$ functions describe a particular kind of gravitational field. It should be stressed that these functions also describe the topological and metrical structural properties of the manifold. Thus, according to general relativity, spacetime must be thought as a structural quality of the field. $g_{i j}$ satisfy a particular law of transformation. Mathematically speaking, they are the components of a tensor (the Riemannian metric tensor) with the property of symmetry, which is preserved in all transformations. It is straightforward to show that these components of the symmetrical tensor describe the gravitational field. The system of differential equations that can be postulated as field law for $g_{i j}$ must confirm that the Ricci tensor must be flat. Equation (3) holds only in vacuum. In other words, when dealing with the vacuum case, the Einstein field equations reduce to the Ricci tensor $R_{i j}=0$.

$$
R_{i j}=0,
$$


However, the fact that there might (and in fact do) exist other fields with different masses and other physical properties cannot be explained by symmetrical $g_{i j}$ fields or gravitational fields. Actually, not even the fact that only positive gravitational masses exist can be deduced from this theory (see [31,51-53]). Therefore, a complete relativistic field theory must be based on a field of a more complex nature, that is, a generalization of the symmetrical tensor field, and hence of Riemannian geometry. Einstein put in a great deal of effort in order to find concepts and general principles that would provide a unitary explanation of the physical world. However, "Einstein never succeeded in constructing a complete system of theoretical physics. The right path, which has led to a more complete unification of physical forces, arose from Hermann Weyl. The main idea of Weyl, that of gauge invariance, was of essential geometrical character. It is clear now that its development allowed a major step in the movement of geometrization of physics, and a deeper comprehension of the mathematical as well physical nature of spacetime" [37]. We now give an overview of this theory (see $[34,35,40,54-56])$ for a detailed historical and mathematical presentation).

As aforementioned, a new advance in the search for an explication of physical forces, essentially of geometrical nature, was accomplished by Hermann Weyl. In 1918-1919 (see $[57,58])$ he outlined what he called a "purely infinitesimal geometry", "which should know a transfer principle for length measurements between infinitely close points only, and which should admit a conformal structure. The allusion is of course to Levi-Civita's parallel displacement principle in a Riemannian manifold embedded in a sufficiently high-dimensional Euclidean space, locally given by:

$$
\xi^{\prime i}=\xi^{i}-\Gamma^{i} j k \xi^{j} d x^{k}
$$

with the $d x^{i}$ to be interpreted as the coordinate representation of a displacement vector between two infinitesimally close points so that the direction vector $\xi^{i}$ has been transferred to $\xi^{\prime i}$. According to Weyl, one has to separate logically the concept of parallel displacement from the metrics and to introduce what he called an affine connection $\Gamma$ on a (differentiable) manifold as a linear torsion-free connection. Thus, Weyl proposes a generalization of Riemannian geometry which seemed to be the most natural mathematical framework for the construction of a unified theory of gravitational and electromagnetic forces (see $[40,59,60])$. This generalized Riemannian metric, a Weylian metric on a differentiable manifold $M$, is given by: (i) a conformal structure on $M$, i.e., a class of (semi-)Riemannian metrics $[g]$ in local coordinates given by $g_{i j}(x)$ or $g_{i j}(x)=\lambda(x) g_{i j}(x)$, with multiplication by $\lambda(x)>0$ (real valued) representing what Weyl considered to be a gauge transformation of the representative of [g], and (ii) a length connection on $M$, i.e., a class of differential forms $\varphi$ in local coordinates represented by $\varphi_{i} d x_{i}, \varphi_{i} d x_{i}-d \log \lambda$-representing the gauge transformation of the representative of $j^{\prime \prime}$ [41]. This new infinitesimal geometry enfolded, in fact, the first formulation of a gauge theory. The idea of gauge was introduced by Weyl in a very influential paper in 1918 [33]).

\section{Symmetry as a Guiding Principle of Spacetime Geometry and Dynamics}

The principle of symmetry states that the laws of nature remain invariant when some transformation is applied to them [61]. For example, with his special principle of relativity (1905), Einstein demonstrated that observers moving with any constant velocity perceive the laws of nature to be the same. There are other spacetime symmetries that tell us that the laws of nature look the same when we rotate or translate our laboratory or reset our clocks. The "standard model" (which corresponds to the group $S U(3) \times S U(2) \times U(1)$ ) is based on a set of symmetry principles, including these symmetries of spacetime and other symmetries that require that the laws of nature take the same form when certain changes are made in the ways that the fields of the theory are labelled (see $[62,63])$. The concept of renormalizability arose from the effort in the late 1940s to make sense of the infinite energies and reaction rates that appeared when calculations in quantum electrodynamics were pushed beyond the first approximation (see $[64,65])$. Renormalization is a procedure 
in quantum field theory by which infinite or divergent parts of the theory can be changed into a finite number of parameters, such as masses and electric charges. This procedure is effective for weak and strong interactions in the standard model. However, it fails for gravity because of the nonlinearities appearing in equations of general relativity.

The difference between Einstein's special theory of relativity (1905) and Lorentz's theory of electrons (1909) can be expressed as follows. The goal of Lorentz's theory of electrons was to show the impossibility of detecting experimentally any motion relative to an immobile æther (in other words, the impossibility of detecting the effects of the motion of matter on the speed of light). Einstein, in contrast, took as a fundamental axiom that the speed of light is the same to all observers. From a modern point of view, what Einstein did was introduce a principle of symmetry-the invariance of laws of nature under changes in the velocity of the observer-as one of the fundamental laws of nature (see [66]). Since Einstein's time, we have become more and more familiar with the idea that symmetry principles of various sorts are legitimate fundamental hypotheses [67]. The "standard model" (which achieved the unification of weak and electromagnetic interactions) is largely based on a set of assumed symmetry principles, and string theory can also be viewed in this way (see $[68,69])$. However, in the days of Lorentz and Einstein, symmetries were generally regarded as mathematical curiosities, of great value to crystallographers but hardly worthy to be included among the fundamental laws of nature. It is not surprising that Lorentz was uncomfortable with Einstein's hypothesis of the principle of relativity.

This is a very important scientific and philosophical point as well, which led to most fundamental developments in past and current attempts to understand the structures of spacetime as well as the ways in which these structures are embodied in physical phenomena.

In Einstein's general relativity theory, the principle of equivalence, a principle of local symmetry (the invariance of the laws of nature under local changes of the spacetime coordinates), dictated the dynamics of gravity, of spacetime itself. As far as the conservation laws are concerned, their significance became evident when, as a result of the interest of Bohr's atomic model, the angular momentum conservation theorem became all-important. However, already in the early 1900s was established the connection between the conservation laws and the fundamental symmetries of space and time (see [67]).

Since the turn of the twentieth century, the connection between conservation laws and invariance principles has been generally accepted. The geometrical principles of invariance were recognized by Poincare first in 1906 [70], so it seemed natural to call the group formed by these invariant properties the Poincaré group. In its generality, the relation between symmetries and conservation laws was established by the German mathematician Emmy Nother (see [71]). Nother's theorem states that any symmetry of the Lagrangian corresponds to a conserved law, and thus, there is a quantity that is constant. For example, if the Lagrangian is independent of the location of the origin of physical system, then that system conserves linear momentum; if it is independent of the base time, then energy is conserved; and if it is independent of the angle of measurement, then angular momentum is conserved.

The true physical meaning and importance of these principles were brought out only by Einstein in his special theory of relativity (as mentioned before). Since displacements in space and time are contained in the group, it can be said that events are correlated, which means that they have the same form independently of where and when they occur; more generally, the laws of nature are similar at any point and at any time in the physical world. In a series of papers on atomic structure and molecular spectra written between 1926 and 1928, E. Wigner laid the foundation both for the application of group theory to quantum mechanics and for the role of symmetry principles in quantum mechanics. Toward the end of the 1930s, he turned his attention to time-dependent symmetries, invariance groups that include time-translation invariance. He considered the Poincaré group [72], which had not been seriously studied previously by either mathematicians or physicists, and posed the question: what are the unitary representations of the Poincare group, and what 
is their physical significance? The answer to these questions consisted in showing that in relativistic quantum mechanics, Poincaré transformations were represented by faithful representations of their covering groups, $\operatorname{ISL}(2, \mathrm{C})$; this allowed him to give a complete classification and an explicit construction of all the irreducible representations. He then provided a definition of an elementary particle, which according to Wigner should be identified as an irreducible representation of the Poincaré group (see [73,74]).

The great physicist C. N. Yang [75] stressed the fact that the forces between particles fulfill almost all of the macroscopic symmetry principles that hold for the electromagnetic interactions. However, weak interactions violate some of these symmetries. Moreover, some new interactions have found that govern the complex pattern of the mutual interaction between the particles. Closely following Yang (see also [76]), we can group all these symmetries into the following four classes:

(a) First, there are the spacetime symmetries, which include symmetries resulting from relativistic invariance and symmetries resulting from reflection invariance and timereversal invariance. These symmetries possess a direct geometrical significance, and they are thoroughly incorporated into the theoretical scheme that describes the elementary particles. Nevertheless, we must take into account the possibility of deviations from the spacetime continuum structure.

(b) Second, we have charge conjugation symmetry, which states roughly that a particle and its antiparticle have the same mass as one another but opposite electric charge and differences in internal quantum number (for a more precise definition, see [77]). This is a purely quantum mechanical concept, and no geometrical concepts such as rotational invariance enters its definition. It is related to the Dirac theory of the electron, which can be viewed as a logical consequence of the fusion of quantum theory with the requirement of relativistic invariance.

(c) Third, we have local internal symmetries. Currently, several conserved charges are recognized, but isospin is not among them. Contrarily to global spacetime symmetries, internal symmetries are invariant only in a certain region of spacetime. An internal symmetry is a transformation acting only on the fields, therefore not transforming spacetime points and leaving the Lagrangian and the physical results invariant $[78,79]$. Example of such symmetries are the $U(1)$ gauge symmetry of Maxwell theory and the $S U(3)$ color symmetry of quantum electrodynamics [80].

(d) Finally, we have a class that comprises the symmetry between identical particles and a type of symmetry called crossing symmetry that associates the reaction rates of two processes (for instance, the relations between the amplitudes for the probability of some collisions).

$$
\begin{aligned}
& a+b \rightarrow c+d \\
& a+d \rightarrow c+b
\end{aligned}
$$

According to some physicists [67,75], this kind of symmetry forms a transition between the geometrical and dynamical principles.

To complete this section, let us quote an important passage of [81]: "We notice that as the domain of physics expands, while symmetry principles increase in number, the increase does seem to follow interdependent and related lines. To understand the relationship and the conceptual unification between them is a major challenge. It seems that three related questions in this pattern of symmetry laws are particularly worth studying. The first is that of the symmetry principle that gives rise to isotopic spin conservation. It is not clear at all whether the isotopic spin conservation is a consequence of an isotopic spin space rotation, or if the isotopic spin space must rather be deduced from the conservation law itself. Another fundamental question relates to the violation of some of the symmetry laws in the weaker interactions. It was found in the early sixties that violation of parity conservation, which represents a spacetime symmetry concept, occurs rather generally among the weak interactions. However, this does not explain why weak interactions are less symmetrical than the stronger one. Finally, if we represent $\mathrm{CP}$ (charge conjugation plus 
space inversion) as the operation that corresponds to space inversion, we thus conclude that all interactions satisfy all space and time symmetries properties, i.e., proper Lorentz invariance, space inversion and time reversal invariance."

$\mathrm{CP}$ (charge conjugation and space inversion) is no longer considered an exact symmetry; it is violated in the quark component of the Standard Model [82]. This violation can be experimentally observed in decay and oscillations of $\mathrm{K}$ and $\mathrm{B}$ mesons and is in broad consistency violated with the Cabibbo-Kobayashi-Maskawa (CKM) mechanism (the quark-mixing matrix). Moreover, the CPT theorem implies the violation of time reversal symmetry (T), which has also been experimentally verified (for more details, see [76]).

\section{Nonhomogeneous Cartan's Geometries and Lie Groups}

We now return to the important question of the connection between geometry and groups, or more precisely, between nonhomogeneous geometries and group theory. After Riemann's (and Clifford's) work, mathematicians developed inhomogeneous geometries, in which one assumes that curvature may change from place to place and that fundamentally local symmetries of space exist. Nonetheless, the Lie group still played an important role at the infinitesimal level, because we can assign Euclidean coordinates to the tangent space (see [83]). In the tangent space, infinitesimally, one must compare different points in different places, and this allows for handling different Lie groups. This theory was developed by Elie Cartan (1927) [84], and it represents the basis of modern differential geometry. It was also the mathematical framework on which Einstein's theory of general relativity is based. In the twentieth century, the global level and properties of Lie groups and differential geometry became the most prominent. This really was a fundamental feature of mathematics, and especially of geometry and topology, in the twentieth century. A major development realized by the work of many mathematicians gave information about so-called "characteristic classes" (see [85]), topological invariants combining together three key parts: Lie groups, differential geometry and topology, and of course, the algebra associated with the group itself.

As we already mentioned, E. Cartan introduced the notion of generalized Riemannian geometry, or new geometries, which played with respect to the different Klein geometries the same role that Riemannian geometries play with respect to Euclidean space. One profound philosophical motivation that lay behind the development by Cartan of more general spaces or geometries may have been the fact that the universe appears to be a subtle mixture of homogeneity and nonhomogeneity, of continuity and discreteness. At almost any location and scale, one is likely to see nothing (i.e., homogeneity) except for some concentrations ("lumps") of something at some great distance. If we move to the center of one of these concentrations, the nonhomogeneity may become more apparent; but if we then change our scale, the nonhomogeneity disappears, and we are left with virtual (or illusory) homogeneity again. In a broad sense, this is the fractal (and fuzzy) nature of the universe. Human life on Earth is quite exceptional in this regard. We appear to be located at a position and scale where the nonhomogeneity (and discreteness) of phenomena are quite manifest. Nevertheless, we were not so completely drowned in nonhomogeneity that we were prevented from discovering Euclidean geometry, a totally homogeneous idealization of our situation. Until the middle of nineteenth century, physics was generally regarded as the study of events in the arena (as it were) of Euclidean geometry. Thanks to Einstein's theory of general relativity and gauge theories of electroweak and strong interactions, physics has undergone a new significant movement of geometrization, characterized by the appearance of inhomogeneous and nonlinear geometries. This view is still more apparent in the structures and predictions of superstring theories. In all these theories, geometry sheds its passive appearance as backdrop and assumes increasingly the role of an actor. Indeed, the question arises: "Is geometry in its various forms the only actor?" (on this subject, see $[86,87])$.

Cartan's new conceptions of space and its further generalizations contributed in a very fundamental way to the clarification of this question, which is at the core of any 
understanding of reality. If Klein geometries represent perfect homogeneity, then Cartan geometries represent a perfect mixture of homogeneity and nonhomogeneity. Riemannian geometry can be regarded as a nonhomogeneous version of Euclidean space. From the viewpoint of physics, Cartan adds "lumps" to the Klein geometries and more fundamental structures to the Riemannian one. For the description and understanding of a nonhomogeneous universe, this conceptual change in the geometric theory turned out to be very important. In fact, gauge theories describe fermions ("matter's particles") as complexvalued functions on a principal bundle over spacetime and bosons ("forces" allowing for interactions between particles) as connections on this principal bundle [88] (see below for more details).

There are, in fact, two forms of Cartan geometries, the local form and the global form. The two forms are equivalent only when the model Klein geometry is effective. However, although this is a very important case, it does not exhaust the interesting possibilities. For example, spin geometries are based on ineffective models. The first definition is of local character, and its analytical (and physical) aspect is more apparent. The second definition is global and is called the principal bundle definition. Roughly speaking, it presents a Cartan geometry as a deformation of a Lie group that fixes the cosets of a closed subgroup (see [89]).

In giving the following mathematical results we follow closely our previous work ([90] see also [60]).

Definition 2. A model geometry for a Cartan geometry consists of (i) an effective infinitesimal Klein geometry $(\boldsymbol{g}, \boldsymbol{h})$, (ii) a Lie group $H$ realizing $\boldsymbol{h}$, and (iii) a representation, denoted by Ad: $H \rightarrow$ $G l_{\text {Lie }}(\boldsymbol{g})$, extending $A d_{\boldsymbol{h}}: H \rightarrow G l_{\text {Lie }}(\boldsymbol{h})$.

The kernel $K$ of the representation Ad: $H \rightarrow G l_{\text {Lie }}(g)$ is called the kernel of the model geometry. If $K$ is trivial, the model geometry is called effective. A model geometry is called primitive if the subalgebra $\boldsymbol{h}$ is a maximal subalgebra of $\boldsymbol{g}$. It is called reductive if there is an $H$ module decomposition $\boldsymbol{g}=\boldsymbol{h} \oplus \boldsymbol{p}$.

Definition 3. Consider a model geometry $(\boldsymbol{g}, \boldsymbol{h})$ with group $H$. A Cartan gauge with this model on a smooth manifold $M$ is a pair $\left(U, \theta_{U}\right)$, where $U$ is an open set of $M$ and $\theta_{U}$ is a g-valued 1-form on $U$ satisfying the regularity condition that

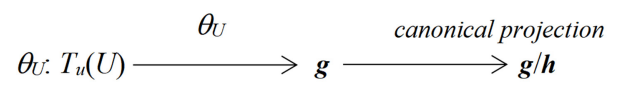

is a linear isomorphism for each $u \in U$.

Definition 4. If $M$ is a smooth manifold, then a Cartan atlas on $M$ is a collection $A=\left\{\left(U_{\alpha}, \theta_{\alpha}\right)\right\}$ of Cartan gauges with model $(\boldsymbol{g}, \boldsymbol{h})$ and group $H$ such that: (i) (covering) the $U_{\alpha} s$ form an open cover of $M$; (ii) (compatibility) if $\left(U, \theta_{U}\right),\left(V, \theta_{V}\right) \in A$, then there exists a smooth map $k: U \cap V \rightarrow H$ such that $\theta_{V}=A d\left(k^{-1}\right), \theta_{U}+k \cdot \omega_{H}$ on $U \cap V$.

One of Cartan's fundamental insights is that for any "Cartan gauge" $\left(U, \theta_{U}\right)$, the $g$-valued 2-form $\Theta_{U}=d \theta_{U}+1 / 2\left[\theta_{U}, \theta_{U}\right]$ on $U$ need not vanish. In fact, $\Theta_{U}$ is a measure of the nonhomogeneity - the lumpiness — of a Cartan geometry.

Definition 5. The form $\Theta_{U}=d \theta_{U}+\frac{1}{2}\left[\theta_{U}, \theta_{U}\right]$ on $U$ associated to a Cartan gauge $(\theta, U)$ is called the curvature with respect to this gauge.

Of course, like the gauge $\theta_{U}$, the curvature is not intrinsically defined. Let us see how it transforms as we alter the gauge. 
Lemma 1. Suppose that $\left(\theta_{i}, U\right)$ are Cartan gauges on $U$ for $i=1,2$. Then:

$$
\theta_{1} \Rightarrow{ }_{h} \theta_{2} \text { implies } \Theta_{2}=\operatorname{Ad}\left(h^{-1}\right) \Theta_{1} \text {. }
$$

A simple consequence of this is that the vanishing of the curvature is an intrinsic condition, independent of the choice of the gauge.

Definition 6. A Cartan geometry is said to be flat if its curvature vanishes at every point.

A local Klein geometry is an example of a Cartan geometry. A smooth Klein geometry can be defined as a manifold $Y$ endowed with a Lie group $G$ of symmetries acting transitively, such that there is at least one $g \in G$ displacing an object from any point in $Y$ to any other point. Let $\Gamma$ be a subgroup of $G$. The manifold $Y$ can be identified with the coset space $G / H$, referred to as a Klein geometry. Now it can be said that $\Gamma$ acts on $G / H$ by left multiplication as a group of covering transformations. The transformations in $\Gamma$ act as geometric isomorphisms on $G / H$. Recall that if $M$ is a manifold of the same dimension as the Klein geometry $G / H$, then the Cartan geometry on $M$ fulfills two important requirements: (i) a principal bundle $P \rightarrow M$ can be defined on it, and (ii) $P$ has a $g$-valued 1-form, i.e., the Cartan connection (see [91]).

Finally, let us define the notion of a gauge symmetry of a Cartan atlas.

Definition 7. Suppose that $A=\left\{\left(U_{\alpha}, \theta_{\alpha}\right) \mid \alpha \in A\right\}$ is a maximal atlas for a Cartan geometry on the manifold $M$. A gauge symmetry of the Cartan geometry is a permutation $b: A \rightarrow A$ such that (i) $U_{b(\alpha)}$, and (ii) $b$ satisfies the compatibility condition: if $U_{\alpha}=U_{\beta}$ and $\theta_{\alpha} \Rightarrow{ }_{h} \theta_{\beta}$, then $\theta_{b(\alpha)} \Rightarrow \theta_{b(\beta)}$.

\section{Some Mathematical Fundamentals about Compact Lie Groups and Clifford Geometric Algebras}

In this section, we introduce some fundamental facts about the concept of Lie orthogonal groups. This should allow for a better understanding of the notion of spin structures and of the essential geometric character of the link between Clifford algebras and compact Lie groups [89,92]. We then highlight the geometric interpretation of Clifford algebra in terms of its quaternionic and spinorial representations. In fact, such an interpretation is connected with the topology of spheres (see [93]) and to the mathematical structure of quantum field theories and especially to supersymmetry (see [94]). Orthogonal groups and the more general Lie groups have many fundamental relationships with physics. As we have already seen, the rotation and Lorentz groups allow for a deep mathematical description of the special relativity theory. Spinors, which in their most general mathematical form were discovered in 1913 by E. Cartan in his investigations on the linear representations of simple Lie groups and the simple representations of all simple Lie algebras, have played a very important role in the attempts by physicists (especially [95-97]) to elucidate the deep structures of quantum mechanics. Spinors are elements on which the new representation of the orthogonal Lie algebra $g$ operates and that cannot be obtained from representation on the vectors on which $g$ operates by the classical operations of constructing tensor products and decomposing them into simple (or irreducible) representation spaces. The simplest case of a spin representation is that for the orthogonal Lie algebra in three variables; this Lie algebra is well known to be isomorphic to the special unitary Lie algebra on two variables, which shows that it has a faithful representation of degree 2: this is its spin representation. In other words, according to Cartan, spinors provide a linear representation of the group of rotations in a space with any number $n$ of dimensions, each spinor having $2^{v}$ components, where $n=2 v+1$ or $2 v$. Moreover, there is a profound relationship between the geometric origin of spinors, the use of matrices in quantum mechanics, and the property possessed by Clifford algebras of representing rotations in space having any number of dimensions. For the moment, let us remark that the unit quaternions form the group SU(2), which is the double cover of the rotation group $S O(3)$. This makes them suited to the study of rotations 
and angular momentum, particularly in the context of quantum mechanics. In fact, this phenomenon is a special case of the theory of Clifford algebras.

On the contrary, the relevance to geometry of the octanions was quite obscure until 1925, when E. Cartan described "triality" - the symmetry between vectors and spinors in eight-dimensional Euclidean space. There is also a close connection between the principle of triality on the one hand and, on the other hand, the exceptional Jordan algebra of dimension 27 and the five exceptional Lie groups. However, attempts by P. Jordan, J. von Neumann, and E. Wigner in the 1930s to apply octanionic quantum mechanics to nuclear and particles physics were rather ineffective. Only recently, around the 1980s, was it realized that the octanions explain some peculiar features of string theory (see [98]). The Lagrangian for the classical superstring involves a relationship between vectors and spinors in Minkowski spacetime that holds only in 3, 4, 6, and 10 dimensions. Note that these numbers are two more than the dimensions of the spaces of $\mathbf{R}, \mathbf{C}, \mathbf{H}$, and $\mathbf{O}$. As Baez and Muniain have remarked (1994) [99], this fact is likely no coincidence, for the isomorphisms sl $(2, \mathbf{R}) \cong s o(2,1), s l(2, \mathbf{C}) \cong s o(3,1)$, sl $(2, \mathbf{H}) \cong s o(5,1)$, and $s l(2, \mathbf{O}) \cong s o(9,1)$ allow treating a spinor in one of these dimensions as a pair of elements of the corresponding division algebra. It is interesting that of these Lagrangians, the 10-dimensional octanionic one provides the most promising framework for a realistic theory of fundamental physics.

\section{Topology and Quantum Field Theory}

Beyond the single cylindric hole or flux tube of the Aharonov-Bohm experiment, we can consider a complicated knotted configuration of tubes (see [100]). The study and classification of such knots is a typical and difficult problem in topology. The more elaborate the knot, the more intricate the structure of the external vacuum is. In fact, over the past twenty years, we have come to see that the topology of knots is intimately related to physics - both macroscopic and microscopic (see [65,101-110]) — and that the formal apparatus of quantum field theory can be used to solve difficult topological problems in the theory of knots. These developments strongly suggest that topological aspects of three-dimensional space, as manifested by knots, should play some fundamental role in quantum physics. Moreover, this role should involve the basic physical framework prior to the introduction of matter or force, in other words, "the vacuum". The relation between the topology of knots and quantum field theory was elaborated in the late 1980s $([42,85,104]$; see also [99]), and the different theories accounting for this relation can be described generically as "topological quantum field theories." The essential characteristic of such theories is that they have the formal structure of a quantum theory (e.g., dealing with discrete spaces and probabilities), but the information they produce is purely topological (e.g., information about the nature of a knot). Differently stated, a topological field theory is independent of the metric $g_{\mu v}$ of the manifold on which it is defined (see [105]). There is no measurement or genuine dynamics in such theories; they deal with nature at a more primordial level. At a later stage, one can imagine superimposing a more conventional physical theory onto the topological background (see, for example, [106]).

The point of departure in Witten's work was the beautiful results by Vaughan Jones (1987) [107], who found many new invariants of knots. Essentially, Jones first drew the knot in the plane as the closure of a braid. To compute the invariants, he applied a complicated formula that depended on the precise form of the braid. The result was an invariant for knots. The key point was that a knot is the closure of many different braids. Jones's formula depended on choosing an arbitrary braid to represent the knot. Thus, the formulae were really formulae for braids that happened to yield the same answer on all braids representing the same knot. It is therefore difficult to see which properties of the knot itself are actually expressed by these formulae.

For several years, finding an intrinsic, geometric definition of the Jones polynomials, without referring to braids, was an unsolved problem. This problem was resolved by Witten, who described a quantum field theory for a 3-manifold embracing knots. The theory had no unnecessary ingredients; only the ambient 3-manifold and an oriented 
collection of knots were given. Witten then produced many significant invariants, all resulting from a path integral over fields. Conformal field theory allows for a careful analysis of the properties of such integrals, and Witten showed that the invariants should coincide with the Jones polynomials. Because Witten's theory was intrinsic, it lent itself to generalizations. Probably the most profound of these generalizations was that he could define invariants for knots in arbitrary 3-manifolds, where the concept of the braid is not in play.

Another (actually the first) topological quantum field theory was not that related to knots in three-dimensional spaces, but a more intricate one related to the deep mathematical results of Simon Donaldson (1983) [41] on four-dimensional geometry, the physical significance of which Was then elucidated by Witten. The Donaldson-Witten theory entailed vacuum solutions. To begin our discussion of four-dimensional space, let us pick a four-dimensional manifold $X$ bearing a metric. Instead of looking at Maxwell fields, we look for gauge fields on $X$ that satisfy the Yang-Mills equations. The Yang-Mills equations are analogous to the Maxwell equations in cases where one studies gauge fields describing the weak or strong interaction. Such fields on $X$ are called vacuum solutions, much in the spirit of the classical vacuum discussed above. A vacuum solution has a quantized charge $k=0,1, \ldots, n$. If we fix the charge, we find a complicated space of solutions called the Yang-Mills moduli space. Because this space depends on $X$ and the metric on $X$, it is not yet a topological invariant of $X$. The moduli space presents all kinds of loops and holes. Some holes in this space disappear if we vary the metric on $X$, while others persist. Clearly, the holes that persist must be (differential) topological invariants of $X$.

Donaldson's remarkable observation (1983) [41] was that such invariant loops do exist, and that they contain deep information about the 4-manifold. Donaldson's theory used classical vacuum to say something about pure space. It is a very powerful theory that shows, for example, that even on good old four-dimensional Euclidean space, there are infinitely many inequivalent ways of defining the differentiability of functions; that is, the differential topology of four-dimensional vector spaces is highly nontrivial.

Witten developed a complicated supersymmetric quantum field theory such that the expectation values of certain observables give the relevant information about persisting loops. Witten's quantum field theory, in its original version, depended on a metric on $X$, just as Donaldson's theory does. He showed that his expectation values do not change if we vary the metric, thereby demonstrating that they are topological invariants-that is, invariants of pure space. However, his theory is full of all kinds of fields and superfields. So again, a nonvacuous theory gives information about the ultimate vacuum, pure space.

In gauge theory, the issue is to find anti-self-dual connections on some bundle over the 4-manifold $X$. These are precisely the connections in which the self-dual part of the curvature vanishes. Thus, we are looking for zeroes of a function $s$ defined on the space $A$ of connections; the function assigns to a connection the self-dual part of its curvature, which is an element of an infinite-dimensional vector space $V$. Gauge invariance enters the picture in the following way. If we apply a gauge transformation to the connection, then the self-dual part of the curvature is conjugated by this gauge transformation. Thus, the function $s$ does not descend to a function on the quotient space $A / G$ of connection modulo gauge transformations, since its values get twisted in $V$. Instead, the function becomes a section of a vector bundle $W$ over $A / G$. Looking for anti-self-dual connection modulo gauge transformations amounts to looking for the zeroes of a section of this bundle.

We now assume that there are only finitely many zeroes in these sections. In practice, this situation is not always met, but it does happen in interesting situations. Donaldson's invariant now obtained simply by counting the number of zeroes, that is, the number of anti-self-dual connections, counted with signs. As indicated below (Figure 1), the nontriviality of a bundle can force a section to have zeros. Generally, the number of zeroes of a section of a bundle $E$ over a compact manifold $M$ is an invariant of $E$, called the Euler number. Notably, it is independent of that section. The Euler number can be computed in 
various ways, and this is precisely the distinction between Donaldson's theory and the Witten-Donaldson theory.

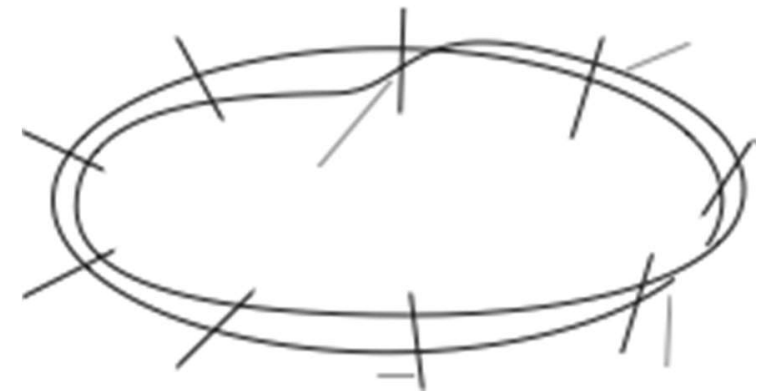

Fibers of Möbius bundle
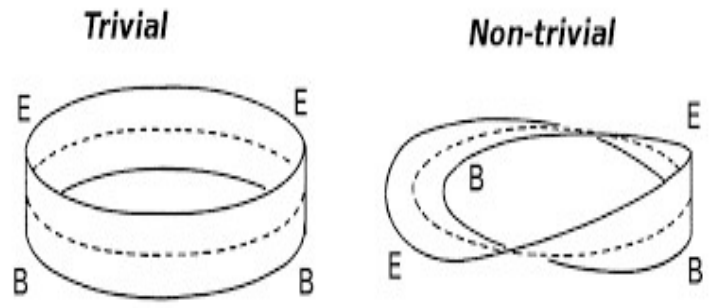

Base space: the circle

Figure 1. (Left) Möbius bundle with a section; (Right) a trivial and a nontrivial bundle.

First of all, one can simply compute the number of zeros in a section; in the case at hand, this amounts to solving the self-dual Yang-Mills equations. A more sophisticated approach is to integrate a differential form over the total space of $E$. The form is the square of the Thom class of the bundle. In the Yang-Mills case, we integrate the square of the Thom class over the infinite-dimensional bundle $W$ over an infinite-dimensional space A/G. Such integrals are normally called Feynman path integrals [108]. This is more or less what Witten did to compute Donaldson's polynomial invariants.

We would like to make another remark relating to recent works on the connection between knot topology and quantum chromodynamics in vacuum. First, we remind the reader that the non-Abelian gauge theory is well known to have a nontrivial topology. In particular, the theory offers infinitely many topologically distinct vacua that can be connected by vacuum tunneling through the instantons (see [106]). The existence of both topologically distinct vacua and vacuum tunneling has played a very important role in quantum chromodynamics (QCD) (see $[109,111])$. Concerning the relation between vacuum and nontrivial topologies, it is here worth mentioning the so-called "boson method" (see [112-115]]). The boson transformation method describes how, when spontaneous symmetry breaking occurs, topological defects can emerge after an inhomogeneous Goldstoneboson condensation on quantum vacuum $[116,117]$. In other words, these defects emerge as macroscopic objects (inhomogeneous condensate) from quantum dynamics. An important result states that only massless bosons are subjected to the boson transformation function bearing topological singularities (for a very good account, see [118]).

The Skyrme theory states that there exists a knot that is topologically stable. In fluid mechanics, this knot behaves as a twisted magnetic vortex ring composed of helical baby skyrmion, which can be understood in terms of topological solitons in a (2+1)-dimensional field theory. Very interestingly, this knot has been shown to describe topologically distinct QCD vacua. This finding is puzzling, because the knot is a physical object that carries a nonvanishing energy. It may appear strange that the knot can be related to a QCD vacuum, but the Skyrme theory itself is closely related to QCD, and both the knot and the QCD vacuum are described by the same topology $\pi_{3}\left(S^{3}\right)$ [119]). As was stressed by [120], "In this circumstance, one needs to know in exactly what sense the QCD vacuum can be identified as the knot. Since there exists one knot solution for each topological quantum number (up to the trivial spacetime translation and the global $S U(3)$ rotation), one might suspect that the knot equation could be viewed as a gauge condition for the topologically equivalent vacua."

In fact, it has been suggested that the knot equation can be viewed as a nonlocal gauge condition that describes the maximal Abelian gauge in SU(2) QCD [121]. More recently, it has been shown that the knot equation is nothing but a generalized Lorentz gauge condition that selects one representative vacuum for each class of topologically equivalent $Q C D$ vacua. 
This conclusion allows interpreting the knot as a complex vector field that couples to an Abelian gauge field and the knot equation as an Abelian gauge condition for the complex vector field (Cho 2007). One of the most important results obtained by Cho was using the Abelian Chern-Simons index of the magnetic potential $C_{\mu}$ to give the knot quantum number through the expression:

$$
Q=g^{2} / 32 \pi^{2} \int \varepsilon_{i j k} C_{i} H_{i k} d^{3} x,
$$

which describes the nontrivial topology of the Hopf mapping $\pi_{3}\left(S^{2}\right)$ defined by $n^{\wedge}$ (in the $\operatorname{SU}(2) \mathrm{QCD}, n^{\wedge}(i=1,2,3)$ is a righthanded local orthonormal frame).

The preimage of the mapping from the compactified space $S^{3}$ to the target space $S^{2}$ defined by $n^{\wedge}$ forms a closed circle, and two preimages of the mapping are linked together when $\pi_{3}\left(S^{2}\right)$ is nontrivial. This linking number is given by the Abelian Chern-Simons index. It is possible to show that the same description applies to the QCD vacuum. In particular, this means that with the same index, the QCD vacuum can also be classified by an Abelian gauge potential. Conversely, with the vacuum equation:

$$
D_{\mu} \omega_{v}-D_{\nu} \omega_{\mu}=0, H_{\mu \nu}=\partial_{\mu} C_{v}-\partial_{\nu} C_{\mu}=i g\left(\omega_{\mu} * \omega_{\nu}-\omega_{\nu} * \omega_{\mu}\right)
$$

where $D_{\mu}$ is the vacuum potential and $\omega_{\mu}$ is a complex vector field), one can transform the knot quantum number above to a non-Abelian form:

$$
Q=g^{2} / 32 \pi^{2} \int \varepsilon_{i j k} C_{i} H_{i k} d^{3} x,=-g^{2} / 32 \pi^{2} \int \varepsilon_{a b c} C_{i}^{a} C_{j}^{b} C_{k}^{c} d^{3} x
$$

This proves that the knot quantum number can be interpreted in terms of a nonAbelian Chern-Simons index. More meaningfully, this tells that the Abelian and nonAbelian Chern-Simons indexes can essentially be deemed mathematically and physically similar.

\section{Conclusions}

Let us conclude with a few general philosophical remarks and then some more specific reflections. It is well known that in field theory dealing with forces such as gravitation or electromagnetism, empty space-the vacuum-alters its nature in the presence of the relevant force. This alteration takes the form of a geometrical distortion that changes the trajectory of any material object that enters the appropriate position of space. This is what we might call the classical theory of the vacuum, classical in the sense of pre-quantum theory. However, as we already stressed, quantum theory requires that we modify our physical ideas in a profound way, and we must inevitably start by reexamining the classical vacuum (see $[55,122,123])$. Quantum field theory attempts to deal with the classical force-fields in a quantum-mechanical way, and the quantum vacuum that emerges from this theory is a complex and mysterious structure that stretches mathematics to its utmost limits (see [88]). Quantum fields fluctuate and convert themselves into particles in a bewildering manner, indicating in particular the fact that the conventional separation between force and matter cannot be maintained (see [124,125]). Still more important (and more surprising) is the fact that the quantum vacuum does have interesting geometrical features, but these relate not to the traditional geometry of Euclid, Riemann, etc., involving measurement, but to that modern branch of the subject known as topology, which is concerned with qualitative properties of space. Unlike measurement, which can be conducted on a small, local scale, topological features are visible only on a "global" scale (see [126]).

Finally, let us mention the idea developed recently by different authors that even neutrino mixing could originate from a topologically nontrivial spacetime (D0 branes in string theory). This produces a nontrivial vacuum structure (flavor vacuum) and the spontaneous symmetry breaking (SSB) of Poincaré symmetry (see [127-131]). 
This relation between topology and the quantum fields has been recognized quite recently, and the full implications of this relationship have been explored in just the last four decades. It is clear that a deeper understanding of the universe rests on the possibility of further elucidating the profound connection between geometry and physics. Let us briefly mention three examples that illustrate how topology deeply relates to quantum theory: (1) the Aharonov-Bohm effect (1959) shows that, even in a force-free region, there are physical effects that are quantum mechanical, inasmuch as they correspond to phase shifts in the wave function of the electron, and they are topological in origin, since there is a cylindrical hole in the force-free region; (2) Dirac's theoretical prediction of the magnetic monopole necessarily implies that both electric and magnetic charges are quantized ([96]; see also [132] for a recent and detailed presentation); (3) entangled topological phases of matter in low dimensions might be deeply related to time-reversal symmetries (see $[55,133])$.

It is likely that in the near future, many other phenomena at the macroscopic (largescale), mesoscopic (supramolecular), and microscopic (quantum-mechanical) levels of the physical world will be understood as emerging from geometrical properties and topological effects. This might lead to discovering new types of symmetries combining local and global rules of transformations and invariance.

Funding: This research received no external funding.

Acknowledgments: We are grateful to the referees for their valuable comments.

Conflicts of Interest: The author declare no conflict of interest.

\section{References}

1. Weyl, H. Symmetry; Princeton University Press: Princeton, NJ, USA, 1983.

2. Plato. Timaeus (Dialogue); Lamb, W.R.M., Translator; Harvard University Press: Cambridge, MA, USA, 1925 ; Volume 9.

3. Galilei, G. Dialogue Concerning the Two Chief World Systems, Ptolemaic and Copernican; University of California Press: Berkeley, CA, USA, 1967.

4. Kepler, J. The Harmony of the World, 1st ed.; American Philosophical Society: Philadelphia, PA, USA, 1997.

5. Zhang, P.M.; Cariglia, M.; Elbistan, M.; Gibbons, G.W.; Horvathy, P.A. Kepler Harmonies and Conformal Symmetries. Phys. Lett. B 2019, 792, 324-328. [CrossRef]

6. Gross, J.D. Symmetry in Physics: Wigner's Legacy. Phys. Today 1995, 48, 46-50. [CrossRef]

7. Wilczek, F. In search of symmetry lost. Nature 2005, 433, 239-247. [CrossRef]

8. Zee, A. Fearful Symmetry. The Search for Beauty in Modern Physics, 2nd ed.; Princeton University Press: Princeton, NJ, USA, 2007.

9. Brading, K.A.; Castellani, E. (Eds.) Symmetries in Physics: Philosophical Reflections; Cambridge University Press: Cambridge, UK, 2003.

10. Coleman, S. Secret symmetry: An introduction to spontaneous symmetry breakdown and gauge fields. In Laws of Hadronic Matter; Zichichi, A., Ed.; Academic Press: New York, NY, USA, 1975; pp. 138-215.

11. Coleman, S. Aspects of Symmetry, Selected Erice Lectures; Cambridge University Press: Cambridge, UK, 1985.

12. Fröhlich, J. Spontaneously broken and dynamically enhanced global and local symmetries. In Non-Perturbative Quantum Field Theory (Selected Papers of Jürg Fröhlich); World Scientific: London, UK, 1992; pp. 193-212.

13. Connes, A. Symétries. Pour La Sci. 2001, 292, 36-43.

14. Connes, A.; Gawedzki, K.; Zinn-Justin, J. (Eds.) Quantum Symmetries/Symétries Quantiques; Elsevier Science \& Technology: Amsterdam, The Netherlands, 1998; Volume 64.

15. Witten, E. When symmetry break down. Nature 2004, 429, 507-508. [CrossRef]

16. Haq, S.; Liu, N.; Humblot, V.; Jansen, A.P.J.; Raval, R. Drastic symmetry breaking in supramolecular organization of enantiomerically unbalanced monolayers at surfaces. Nat. Chem. 2009, 1, 409-414. [CrossRef] [PubMed]

17. Drienfel'd, V.G. Quantum Groups. In Proceedings of the International Congress of Mathematicians, Berkeley, CA, USA, 3-11 August 1986; American Mathematical Society: Berkeley, CA, USA; pp. 798-820.

18. Nakagawa, K.; Sen, R.N.; Umezawa, H. Dynamical rearrangement of symmetries. IL Nuovo Cim. A 1966, 42, 565-588. [CrossRef]

19. Matsumoto, H.; Umezawa, H.; Vitiello, G.; Wyly, J.K. Spontaneous breakdown of a non-Abelian symmetry. Phys. Rev. D 1974, 9 , 2806-2813. [CrossRef]

20. De Concini, C.; Vitiello, G. Spontaneous breakdown of symmetry and group. Nucl. Phys. B 1976, 116, 141-156. [CrossRef]

21. Celeghini, E.; Magnollay, P.; Tarlini, M.; Vitiello, G. Nonlinear realization and contraction of group representations. Phys. Let. B 1985, 162, 133-136. [CrossRef]

22. Penrose, R. Structure of Space-Time. In Battelle Rencontres; Lectures in Mathematics and Physics; DeWitt, C.M., Wheeler, J.A., Eds.; Benjamin: New York, NY, USA, 1967; pp. 121-235.

23. Hawking, S.W.; Penrose, R. The Nature of Space and Time; Cambridge University Press: Cambridge, UK, 1994. 
24. Boi, L. Theories of Space-Time in Modern Physics. Synthese 2004, 137, 36-88. [CrossRef]

25. Stachel, J. History of relativity. In Twentieth Century Physics; Brown, L., Pais, A., Pippard, B., Eds.; Springer: New York, NY, USA, 1995; pp. 249-356.

26. Torretti, R. Relativity and Geometry; Dover: New York, NY, USA, 1996.

27. Mehra, J. Einstein, Hilbert and the Theory of Gravitation. Historical origins of General Relativity Theory; Reidel: Dordrecht, The Netherlands, 1974.

28. Boi, L. Le Problème Mathématique de L'espace. Une Quête de L'intelligible; Thom, R., Ed.; Springer: Heidelberg/Berlin, Germany, 1995.

29. Cartan, E. Sur les variétés à connexion affine et la théorie de la relativité généralisée. Ann. Sci. L'école Norm. Supérieure 1923, 40, 325-412. [CrossRef]

30. Hehl, F.W.; Von der Heyde, P.; Kerlick, G.D.; Nester, J.M. General relativity with spin and torsion: Foundations and prospects. Rev. Mod. Phys. 1976, 48, 393-416. [CrossRef]

31. Regge, T. Physics and Differential Geometry. In 1830-1930: A Century of Geometry. Epistemology, History and Mathematics; Boi, L., Flament, D., Salanskis, J.-M., Eds.; Lecture Notes in Physics; Springer: Berlin/Heidelberg, Germany, 1992.

32. Trautman, A. On the structure of Einstein-Cartan equations. Symp. Math. 1973, 12, 139-154.

33. Weyl, H. Reine Infinitesimalgeometrie. Math. Zeit. 1918, 2, 384-411. [CrossRef]

34. Lubkin, E. Geometric Definition of Gauge Invariance. Ann. Phys. 1963, 23, 233-283. [CrossRef]

35. O'Raifeartaigh, L. The Dawning of Gauge Theory; Princeton University Press: Princeton, NJ, USA, 1997.

36. Redhead, M.L.G. The interpretation of gauge symmetry. In Ontological Aspects of Quantum Field Theory; Kuhlmann, M., Lyre, H., Wayne, A., Eds.; World Scientific: Singapore, 2003.

37. Boi, L.H. Weyl's deep insights into the mathematical and physical world. His important contribution to the philosophy of space, time and reality. In Weyl and the Problem of Space; Lobo, C., Julien, B., Eds.; Springer: Berlin/Heidelberg, Germany, 2019; pp. 231-263.

38. Wu, T.T.; Yang, C.N. Concept of nonintegrable phase factors and global formulation of gauge fields. Phys. Rew. D 1975, 12, 3845-3857. [CrossRef]

39. Weyl, H. The Theory of Groups and Quantum Mechanics; Princeton University Press: Princeton, NJ, USA, 1931.

40. Bourguignon, J.-P.; Lawson, H.B., Jr. Yang-Mills Theory: Its Physical Origins and Differential Geometric Aspects. In Seminar on Differential Geometry; Yau, S.-T., Ed.; Annals of Mathematical Studies No. 102; Princeton University Press: Princeton, NJ, USA, 1982; pp. 395-421.

41. Donaldson, S.K. An Application of Gauge Theory to Four-Dimensional Topology. J. Diff. Geom. 1983, 18, 279-315. [CrossRef]

42. Atiyah, M.F. Topological Quantum Field Theories. Publications Mathématiques de l'IHÉS 1988, 68, $175-186$.

43. Kobayashi, S.; Nomizu, K. Foundations of Differential Geometry; Wiley-Interscience: New York, NY, USA, 1969 ; Volume 2.

44. Spivak, M. A Comprehensive Introduction to Differential Geometry, 3rd ed.; Publish or Perish: Los Angeles, SA, USA, 1999.

45. Sharpe, R.W. Differential Geometry. Cartan's Generalization of Klein's Erlangen Program; Springer: New York, NY, USA, 1997.

46. Gromov, M. Sign and Geometric Meaning of Curvature. Rend. Mat. Dell'univ. Bologna 1991, 61, 9-123. [CrossRef]

47. Schoen, R.; Yau, S.-T. Lectures on Differential Geometry; Conference Proceedings and Lectures Notes in Geometry and Topology; International Press: Cambridge, MA, USA, 1994; Volume 1.

48. Morgan, J.W.; Tian, G. Ricci Flow and the Poincaré Conjecture; American Mathematical Society: Providence, RI, USA, 2007.

49. Einstein, A. Die Grundlagen der allgemeinen Relativitätstheorie. Ann. Phys. 1916, 49, 769-822. [CrossRef]

50. Einstein, A. Fundamental Ideas and Problems of the Theory of Relativity; Elsevier: Amsterdam, The Netherlands, 1923.

51. Casimir, H.B.G. On the attraction between two perfectly conducting plates. Proc. Koninkl. Ned. Akad. Wet. 1948, 51, 793-796.

52. Boi, L. Creating the World ex nihilo? On the Quantum Vacuum and Its Fluctuations. In The Two Cultures: Shared Problems; Carafoli, E., Danieli, G.A., Longo, G.O., Eds.; Springer: Milan, Italy, 2009; pp. 51-97.

53. Sitenko, Y.A. Vacuum Polarization Effects in the Background of Nontrivial Topology; Lecture Notes in Physics; Springer: Berlin/Heidelberg, Germany, 2000; Volume 543.

54. Kibble, T.W.B. Geometrization of Quantum Mechanics. Commun. Math. Phys. 1979, 65, 189-201. [CrossRef]

55. Penrose, R. The Road to Reality: A Complete Guide to the Laws of the Universe; Vintage: London, UK, 2004.

56. Katz, S.; Vafa, C. Matter from geometry. Nucl. Phys. B 1997, 497, 146-154. [CrossRef]

57. Weyl, H. Gravitation und Elektrizität. Sitzungsber. Preuss. Akad. Berl. 1918, 26, 465-480.

58. Weyl, H. Eine neue Erweiterung der Relativitätstheorie. Ann. Phys. 1919, 59, 101-133. [CrossRef]

59. Bourguignon, J.-P. Transport parallèle et connexions en géométrie et en physique. In 1830-1930: A Century of Geometry; Springer: Berlin/Heidelberg, Germany, 1992.

60. Boi, L. Geometrical and Topological Foundations of Theoretical Physics: From Gauge Theories to String Program. Inter. J. Math. Mathem. Sci. 2004, 34, 1777-1836. [CrossRef]

61. Poincaré, H. La Science et l'Hypothèse; Flammarion: Paris, France, 1902.

62. Sakurai, J.J. Symmetry Laws and Elementary Particles Interactions. In Lectures in Theoretical Physics; Brittin, W.E., Downs, B.W., Eds.; Interscience: New York, NY, USA, 1960; pp. 31-209.

63. Salam, A. Gauge unification of fundamental forces. Rev. Mod. Phys. 1980, 93, 525-536. [CrossRef]

64. Schwinger, J. Quantum electrodynamics. I. A covariant formulation. Phys. Rev. 1948, 74, 1439-1461. [CrossRef] 
65. Boi, L. The Quantum Vacuum. A Scientific and Philosophical Concept: From Electrodynamics to String Theory and the Geometry of the Microscopic World; Johns Hopkins University Press: Baltimore, MD, USA, 2011.

66. Weinberg, S. Gravitation and Cosmology. Principles and Applications of the General Theory of Relativity; Wiley: New York, NY, USA, 1973.

67. Wigner, E.P. Symmetries and Reflections; Indiana University Press: Bloomington, Indiana; London, UK, 1967.

68. Witten, E. Physics and Geometry. In Proceedings of the International Congress of Mathematicians, Berkeley, CA, USA, 3-11 August 1986; Gleason, A.M., Ed.; American Mathematical Society: Berkeley, CA, USA, 1987; pp. $267-303$.

69. Veneziano, G. Quantum geometric origins of all forces in string theory. In The Geometric Universe: Science, Geometry, and the Work of Roger Penrose; Huggett, S.A., Ed.; Oxford University Press: Oxford, UK, 1998; pp. 235-243.

70. Poincaré, H. Sur la dynamique de l'électron. Rend. Circ. Mat. Palermo 1906, 21, 129-175. [CrossRef]

71. Nother, E. Invariante Variationsproblem. Nachr. König. Gesellsch. Wiss. Zu Göttingen Math.-Phys. Kl. 1918, 2, $235-257$.

72. Wigner, E.P. The unitary representation of the inhomogeneous Lorentz group. Ann. Math. 1939, 40, 149-187. [CrossRef]

73. Gross, D.J. Gauge Theory: Past, Present and Future. In Chen Ning Yang. A Great Physicist of the Twentieth Century; Liu, C.S., Yau, S.-T., Eds.; International Press: Boston, MA, USA, 1995.

74. Straumann, N. Unitary representations of the homogeneous Lorentz Group and their significance in quantum physics. In Springer Handbook of Spacetime; Ashetekar, A., Petkov, V., Eds.; Springer: Berlin/Heidelberg, Germany, 2014; pp. $265-278$.

75. Yang, C.N. Symmetry Principles in Modern Physics. In Selected Papers; Yang, C.N., Ed.; W.H. Freeman: San Francisco, CA, USA, 1983; pp. 267-280.

76. Zuber, J.-B. Invariances in Physics and Group Theory; International Center for Fundamental Physics, UPMC Sorbonne Universités Editions: Paris, France, 2012.

77. Greiner, W.; Reinhardt, J. Field Quantization; Springer: Berlin/Heidelberg, Germany, 1996.

78. Nambu, Y. Dynamical symmetries and fundamental fields. In Symmetry Principles at High Energies; Kursunoglu, B., Pelmutter, A., Sakmar, A., Eds.; Freeman: San Francisco, CA, USA; London, UK, 1965; pp. 274-285.

79. Schwinger, J. On gauge invariance and vacuum polarization. Phys. Rev. 1951, 82, 664-679. [CrossRef]

80. Utiyama, R. Invariant theoretical interpretation of interaction. Phys. Rev. 1956, 101, 1597-1607. [CrossRef]

81. Yang, C.N. Selected Papers (1945-1980) of Chen Ning Yang; World Scientific: Shanghai, China, 2005.

82. Gellman, M. Symmetries of baryons and mesons. Phys. Rev. 1962, 125, 1076-1084.

83. Weyl, H. Theorie der Darstellung kontinuierlicher halbeinfacher Gruppen durch lineare Transformationen. I. II. III. Math. Z. 1925, 23, 271-309.

84. Cartan, E. La géométrie des groups simples. Ann. Mat. 1927, 4, 209-256. [CrossRef]

85. Chern, S.-S.; Simons, J. Characteristic forms and geometric invariants. Ann. Math. 1974, 99, 48-69. [CrossRef]

86. Witten, E. Topological quantum field theory. Commun. Math. Phys. 1988, 117, 353-386. [CrossRef]

87. Vafa, C. Geometric physics. Doc. Math. 1998, I, 537-556, Extra Volume ICM.

88. Manin, Y.I. Gauge Field Theory and Complex Geometry; Springer: Berlin/Heidelberg, Germany, 1988.

89. Borel, A. Hermann Weyl and Lie Groups. In Hermann Weyl 1885-1985 Centenary Lectures; Chandrasekharan, K., Ed.; Springer: Berlin/Heidelberg, Germany, 1985; pp. 53-74.

90. Boi, L. Ideas of geometrization, geometric invariants of low-dimensional manifolds, and topological quantum field theories. Int. J. Geom. Methods Mod. Phys. 2009, 6, 1-57. [CrossRef]

91. Kobayashi, S. Theory of Connections. Ann. Mat. Pura Appl. 1957, 43, 119-194. [CrossRef]

92. Boi, L. Clifford geometric algebras, spin manifolds, and group action in mathematical physics. Adv. Appl. Clifford Algebras 2009, 19, 611-656. [CrossRef]

93. Morandi, G. The Role of Topology in Classical and Quantum Physics; Springer: Berlin/Heidelberg, Germany, 1992.

94. Zumino, B. Supersymmetry and the vacuum. Nucl. Phys. B 1975, 89, 535-546. [CrossRef]

95. Pauli, W. Zur Quantenmechanik des magnetischen Elektrons. Z. Für Phys. 1927, 43, 601-623. [CrossRef]

96. Heisenberg, W. Quantum theory of fields and elementary particles. Rev. Mod. Phys. 1957, 29, 269-278. [CrossRef]

97. Dirac, P. Quantized singularities in the electromagnetic field. Proc. Roy. Soc. A 1931, 133, 60-72.

98. Lawson, H.B., Jr. The Theory of Gauge Fields in Four Dimensions; American Mathematical Society: Providence, RI, USA, 1985.

99. Baez, J.; Muniain, J.P. Gauge Fields, Knots and Gravity; World Scientific: Singapore, 1994.

100. Aharonov, Y. Non-local phenomena and the Aharonov-Bohm effect. In Quantum Concepts in Space and Time; Penrose, R., Isham, C.J., Eds.; Clarendon Press: Oxford, UK, 1986; pp. 41-56.

101. Moffat, H.K. The degree of knottedness of tangled vortex lines. J. Fluid Mech. 1969, 35, 117-129. [CrossRef]

102. Boi, L. Symétries, Brisures de Symétries et Complexité, en Mathématiques, Physique et en Biologie; Peter Lang: Berne, Switzerland, 2006.

103. Boi, L. Mathematical knot theory. In Encyclopedia of Mathematical Physics; Françoise, J.P., GTsun, T.S., Eds.; Elsevier: Oxford, UK, 2006; pp. 399-407.

104. Atiyah, M.F. Geometry of Yang-Mills Fields; Lezioni Fermiane, Accademia Nazionale dei Lincei, Edizioni Scuola Normale Superiore: Pisa, Italy, 1979.

105. Beaulieu, L. On the symmetries of topological quantum field theories. Int. J. Mod. Phys. 1995, 10, 4483-4500. [CrossRef]

106. Hooft, G. Topology of the gauge condition and new confinement phases in non-Abelian gauge theories. Nucl. Phys. B 1981, 190, 455-478. [CrossRef] 
107. Jones, V. Hecke Algebra Representations of Braid Groups and Link Polynomials. Ann. of Math. 1987, 126, 335-388. [CrossRef]

108. Feynman, R. The space-time approach to quantum electrodynamics. Phys. Rev. 1949, 76, 769-789. [CrossRef]

109. Wilczek, F. QCD and natural philosophy. Ann. Inst. Henri Poincaré 2003, 4, 211-228. [CrossRef]

110. Polyakov, A.M. Quark confinement and the topology of gauge groups. Nucl. Phys. B 1977, 120, 429-452. [CrossRef]

111. Wilczek, F.; Gross, D.J. Asymptotically free gauge theories. I. Phys. Rev. D 1973, 8, 3633-3652.

112. Leplae, L.; Mancini, F.; Umezawa, H. Boson Method in Superconductivity: Application to the Study of Vortex Lines. Phys. Rev. B 1970, 2, 3594-3605. [CrossRef]

113. Umezawa, H. Advanced Field Theory: Micro, Macro, and Thermal Physics; AIP Press: New York, NY, USA, 1993.

114. Blasone, M.; Jizba, P. Topological defects in inhomogeneous condensates in quantum field theory: Kinks in $(1+1) \lambda \psi^{4}$-dimensional theory. Ann. Phys. 2002, 295, 230-260. [CrossRef]

115. Acquaviva, G.; Iorio, A.; Smaldone, L. Topologically inequivalent quantizations. arXiv 2012, arXiv:2012.09929v1.

116. Goldstone, J.; Salam, A.; Weinberg, S. Broken symmetries. Phys. Rev. 1962, 127, 965-970. [CrossRef]

117. Higgs, P.W. Broken symmetries and the mass of gauge bosons. Phys. Rev. Lett. 1964, 13, 508-509. [CrossRef]

118. Blasone, M.; Vitiello, G.; Jizba, P. Quantum Fields with Topological Defects. In Encyclopedia of Mathematical Physics; Francçoise, J.-P., Naber, G., Tsou, S.T., Eds.; Elsevier: Oxford, UK, 2006; Volume 1, pp. 221-229.

119. Skyrme, T.H.R. A non-linear field theory. Proc. Roy. Soc. A 1961, 260, 127-138.

120. Cho, Y.M. Knot topology of QCD vacuum. Phys. Lett. B 2002, 644, 208-211. [CrossRef]

121. Van Baal, P.; Wipf, A. Classical gauge vacua as knots. Phys. Lett. B 2001, 515, 181-184. [CrossRef]

122. Callender, C.; Hugget, N. (Eds.) Physics Meets Philosophy at the Planck Scale; Cambridge University Press: Cambridge, UK, 2001.

123. Hooft, G. The conceptual basis of quantum field theory. In Handbook of the Philosophy of Science, Philosophy of Physics; Gabbay, D.M., Thagard, P., Woods, J., Eds.; Elsevier: London, UK, 2006.

124. Belavin, A.A.; Polyakov, A.M. Quantum fluctuations of pseudoparticles. Nucl. Phys. B 1977, 123, 429-444. [CrossRef]

125. Anderson, A.; DeWitt, B. Does the topology of space fluctuate? In Between Quantum and Cosmos; Studies and Essays in Honor of John Archibald Wheeler; Zurek, W.H., van der Merwe, A., Miller, W.A., Eds.; Princeton University Press: Princeton, NJ, USA, 1988; pp. 74-88.

126. Isham, C.J. Topological and global aspects of quantum field theory. In Relativity, Groups and Topology II; DeWitt, B.S., Stora, R., Eds.; North-Holland: Amsterdam, The Netherlands, 1984; pp. 1059-1290.

127. Mavromatos, N.E.; Sarkar, R.; Tarantino, W. Flavor Condensate in Brane Models and Dark Energy. Phys. Rev. D. 2009, 80, 084046-084061. [CrossRef]

128. Mavromatos, N.E.; Sarkar, R.; Tarantino, W. D-Foam Induced Flavor Condensates and Breaking of Supersymmetry in Free Wess-Zumino fluids. Phys. Rev. D. 2011, 84, 044050-044069. [CrossRef]

129. Mavromatos, N.E.; Sarkar, R.; Tarantino, W. Condensate Structure od D-Brane Defect Induced Flavor Vacuum. Mod. Phys. Lett. A 2013, 28, 1350045-1350062. [CrossRef]

130. Blasone, M.; Jizba, P.; Mavromatos, N.E.; Smaldone, L. Dynamical generation of field mixing via flavor vacuum condensate. Phys. Rev. D 2019, 100, 045027. [CrossRef]

131. Blasone, M.; Jizba, P.; Mavromatos, N.E.; Smaldone, L. Some nontrivial aspects of Poincaré and C P T invariance of flavor vacuum. Phys. Rev. D 2020, 102, 025021. [CrossRef]

132. Atiyah, M.F.; Hitchin, N.J. The Geometry of Magnetic Monopoles; Princeton University Press: Princeton, NJ, USA, 1998.

133. Boi, L. Asymmetries, Irreversibility, and Dynamics of Time. In The Direction of Time; Albeverio, S., Blanchard, S., Eds.; Springer: Berlin/Heidelberg, Germany, 2014; pp. 96-116. 